Classification

Physics Abstracts

62.20D

\title{
Elastic constants of the layer compound GaS
}

\author{
A. Polian $\left({ }^{*}\right)\left({ }^{+}\right)$, M. Grimsditch $\left({ }^{* *}\right)\left({ }^{++}\right)$, M. Fischer $\left({ }^{* * *}\right)$ and M. Gatulle $(* * *)$ \\ (*) Laboratoire de Physique des Solides $\left({ }^{++}\right)$, Université Pierre et Marie Curie, \\ 4, Place Jussieu, 75230 Paris Cedex 05, France \\ $\left.{ }^{(* *}\right)$ Max Planck Institut für Festkörperforschung, Heisenbergstrasse 1, D 7000 Stuttgart 80, Germany \\ $\left({ }^{* * *}\right)$ Département de Recherches Physiques $\left({ }^{+++}\right)$, Université Pierre et Marie Curie, \\ 4, Place Jussieu, 75230 Paris Cedex 05, France
}

(Reçu le 8 mars 1982, révisé le 5 avril, accepté le 15 avril 1982)

\begin{abstract}
Résumé. - Les constantes élastiques du semiconducteur lamellaire GaS ont été mesurées par diffusion Brillouin, diffusion inélastique de neutrons et par une méthode ultrasonore. Les valeurs obtenues par ces diverses méthodes sont cohérentes entre elles, mais sont différentes des valeurs publiées. A partir de nos valeurs, les compressibilités linéaires sont calculées, en bon accord avec les résultats expérimentaux publiés. Le calcul des compressibilités à partir des constantes élastiques a été effectué pour les composés voisins GaSe et InSe. Nos valeurs des compressibilités de GaSe sont plus sûres que la précédente détermination.
\end{abstract}

\begin{abstract}
The elastic constants of the layer semiconductor GaS were measured using Brillouin scattering, inelastic neutron scattering and ultrasonic techniques. The values obtained by these methods agree well with each other, but disagree with previous published values. From our values, the linear compressibilities are deduced and are shown to fit with the published experimental values. The calculation of the compressibilities using published elastic constants is also done for the related compounds $\mathrm{GaSe}$ and InSe. Our values of the compressibilities of $\mathrm{GaSe}$ are shown to be more reliable than the previous determination.
\end{abstract}

1. Introduction. - Layer compounds, whose physical properties may exhibit large anisotropies, consist of layers of tightly bonded atoms, the layers themselves being loosely bonded by Van der Waals forces.

In a recent study of the pressure dependence of the elastic moduli [1] of GaS, we found a discrepancy in the value of $C_{11}$ at atmospheric pressure as compared with previous determinations $[2,3]$. The above discrepancy prompted this investigation of the elastic moduli of $\mathrm{GaS}$.

Gallium sulfide is a hexagonal layer crystal which belongs to the $D_{6 h}^{4}$ space group. Therefore

$\left(^{+}\right)$Present Address : Laboratoire de Physique des Milieux très Condensés, associé au C.N.R.S.; Université Pierre et Marie Curie, 4, Place Jussieu, 75230 Paris Cedex 05, France.

$\left({ }^{++}\right)$Present Address : Argonne National Laboratory, 9700 South Case Avenue, Argonne, Illinois 60439, U.S.A.

$\left(^{++}\right)$Associé au C.N.R.S. 
it is characterized by 5 independent elastic constants $C_{11}, C_{33}, C_{44}, C_{12}$ and $C_{13} . C_{11}, C_{12}$ and $C_{44}$ determine the velocity of the phonons propagating perpendicular to the hexagonal $c$-axis, and $C_{33}$ and $C_{44}$ that of phonons travelling parallel to it. $C_{13}$ alone appears in the expression for the velocity for intermediate propagation directions.

There are three experimental procedures commonly used to determine elastic constants : Brillouin scattering, ultrasonic measurements and inelastic neutron scattering.

In this paper, we present results obtained by the three methods and compare them to earlier results obtained by neutron scattering [2] and Brillouin scattering [3].

2. Experimental. - 2.1 SAMPLes. - The samples used in these experiments were grown either by the Bridgman method (large samples for neutron scattering and ultrasonic measurements) or by transport in iodine vapour (needles for Brillouin scattering) by A. Chevy from the Laboratoire de Luminescence (Université P. et M. Curie).

2.2 Measurements. - Neutron scattering experiments were performed at the C.E.N. Grenoble on the triple axis spectrometer DN1 in collaboration with J. Rossat-Mignod. We measured the dispersion curves in the $\Gamma \rightarrow \mathrm{M}$ and $\Gamma \rightarrow \mathrm{K}+\mathrm{M}$ directions [4] for the longitudinal and the out-of-layer-plane transverse acoustic phonons which yield information on $C_{11}$ and $C_{44}$.

- Brillouin scattering experiments were performed at the Max Planck Institut in Stuttgart. We used the $647.1 \mathrm{~nm}$ line of a krypton laser as the exciting radiation. Various scattering geometries were used in order to determine the five elastic constants.

- Ultrasonic measurements were done at the Département de Recherches Physiques. An interferometric method was used for both longitudinal and transverse phonons. Three different samples were used : one cleaved perpendicular to the $c$-axis (determination of $C_{33}$, and $C_{44}$ ), one cut and polished parallel to the $c$-axis $\left(C_{11},\left(C_{11}-C_{12}\right) / 2\right.$ and $\left.C_{44}\right)$ and one cut and polished at $45^{\circ}$ from the $c$-axis in order to determine a combination involving $C_{13}$.

3. Results and discussion. - 3.1 Elastic constants. - Our results are shown and compared with published values in table $\mathrm{I}$. There is a fairly good agreement for $C_{33}$ and $C_{12}$ between our results and earlier ones. Let us compare in detail the different results for the other constants :

$C_{11}$ : Our results obtained by Brillouin scattering and ultrasonic measurements coincide to within $1 \%$. Our neutron scattering results, intrinsically less precise, also fall within the error

Table I. - Elastic constants in gigapascals measured by various methods. The neutron results [2] were recalculated using the published dispersion curves.

\begin{tabular}{|c|c|c|c|c|c|c|c|}
\hline \multirow{2}{*}{$\begin{array}{c}\text { Elastic constants } \\
(\mathrm{GPa})\end{array}$} & \multicolumn{3}{|c|}{ Neutrons } & \multirow{2}{*}{$\begin{array}{c}\text { Brillouin } \\
{[3]}\end{array}$} & $\begin{array}{c}\text { Ultrasound } \\
{[12]}\end{array}$ & \multicolumn{4}{c|}{ Present work } \\
\cline { 2 - 5 } & Published [2] & Recalculated & & & Neutrons & Brillouin & Ultrasound \\
\hline$C_{11}$ & 155 & 117 & 157 & & 118 & $121 \pm 2$ & $123.3 \pm 0.2$ \\
\hline$C_{33}$ & 36.4 & 37.6 & 35.8 & 38.6 & & $38 \pm 1$ & $38.5 \pm 0.1$ \\
\hline$C_{44}$ & 13.3 & 13.9 & 8.1 & & 12.7 & $9.8 \pm 0.4$ & $9.96 \pm 0.01$ \\
\hline$C_{12}$ & & 37.6 & 33.2 & & & $34 \pm 3$ & $34.6 \pm 0.3$ \\
\hline$C_{13}$ & & & 15 & & & $11 \pm 3$ & $12.5 \pm 2.5$ \\
\hline
\end{tabular}


bars. The value of $C_{11}$ obtained by these three methods ( $\left.\sim 122 \mathrm{GPa}\right)$ is in disagreement with the values given in references [2] and [3] (i.e. $~ 155 \mathrm{GPa})$. When it is derived from neutron scattering data, $C_{11}$ is given by the initial slope of the dispersion curve of the LA branch in the $\Gamma \rightarrow \mathrm{M}$ (and/or in the $\Gamma \rightarrow \mathrm{K}$ ) direction. Using the experimental dispersion curve given in reference [2], we found a value of $117 \mathrm{GPa}$ which is in good agreement with our results, and not $155 \mathrm{GPa}$ which is given in [2] probably by error, since when we recalculate $C_{33}$ and $C_{44}$ we get the same results as quoted in [2]. Moreover we were able to obtain $C_{12}$ from the dispersion curves of reference [2]. On the other hand, we are at loss to give an explanation for the value given in reference [3]. We propose therefore for $C_{11}$ a value of $123.3 \pm 0.3 \mathrm{GPa}$.

$C_{44}$ : For this constant, there are two sets of results : on the one hand, the Brillouin and ultrasound results and on the other, the neutron results. The discrepancy between these two groups can be explained in the following way : $C_{44}$ is determined from the sound velocity of the transverse acoustic $B_{1}$ phonon propagating in the $\Gamma \rightarrow M$ direction. In this direction, in layer compounds, the dispersion of this TA mode is not linear in the wave vector, but shows a noticeable quadratic dependence [2] i.e. :

$$
v^{2}=\alpha^{2} \zeta^{2}+\beta^{2} \zeta^{4}
$$

where $v$ is the frequency in $\mathrm{Hz}, \zeta=\frac{a \sqrt{3}}{4 \pi} q$ (a lattice parameter and $q$ wave vector), $\alpha=\frac{2}{a \sqrt{3}}\left(\frac{C_{44}}{\rho}\right)^{1 / 2}(\rho$ density $)$ and $\beta$ is a constant [5].

$C_{44}$ is determined from the slope of the dispersion curve extrapolated to zero wave vector. Due to the fact that the Bragg reflexion peaks prevent neutron measurements at very small $q$ values, and because of the parabolic nature of the dispersion curve, the value obtained for $C_{44}$ can only be interpreted as an upper value. This explains the larger value of $C_{44}$ found by this method.

$C_{13}$ : This elastic constant does not appear in any of the expressions for the velocity along high symmetry directions. It appears only for propagation directions between [001] and [100] as given in reference [6].

$$
\begin{aligned}
v_{ \pm}(\theta)= & \frac{1}{\sqrt{\rho}}\left\{\frac{1}{2}\left(C_{44}+C_{11} \sin ^{2} \theta+C_{33} \cos ^{2} \theta\right) \pm\left[\left(C_{44}+C_{11} \sin ^{2} \theta-C_{33} \cos ^{2} \theta\right)^{2}-\right.\right. \\
& \left.\left.-4 C_{44} \sin ^{2} \theta\left(C_{11} \sin ^{2} \theta-C_{33} \cos ^{2} \theta\right)+C_{13}\left(C_{13}+C_{44}\right) \sin ^{2} 2 \theta\right]^{1 / 2}\right\}^{1 / 2}
\end{aligned}
$$

where $\theta$ is the angle between the phonon propagation direction and the $c$-axis.

In figure 1 we present the measured values of $v^{2} \rho$ versus $\theta$ as determined by Brillouin and ultrasonics. By using the values of $C_{11}, C_{12}, C_{33}$ and $C_{44}$ determined along high symmetry directions, we can fit the experimental data according to equation (2) by adjusting $C_{13}$. It is clear that this determination must take into account the uncertainties on the other elastic constants and on the angle $\theta$. The uncertainty on $C_{13}$ is thus of the order of $25 \%$ which tenders consistent all values in table I, within experimental error.

3.2 COMPRESSIBILITIES. - In hexagonal crystals, the elastic constants and the linear compressibilities are related by :

$$
\begin{aligned}
& \chi_{\|}=\frac{C_{11}+C_{12}-2 C_{13}}{C_{33}\left(C_{11}+C_{12}\right)-2 C_{13}^{2}} \\
& \chi_{\perp}=\frac{C_{33}-C_{13}}{C_{33}\left(C_{11}+C_{12}\right)-2 C_{13}^{2}}
\end{aligned}
$$




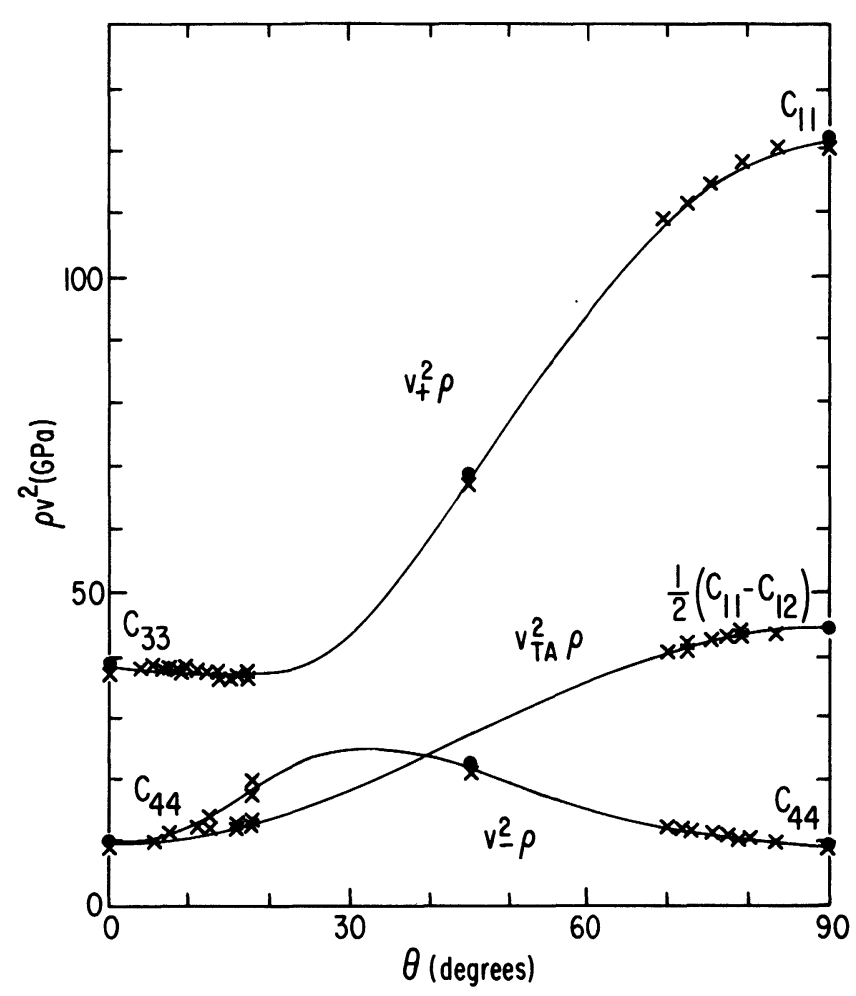

Fig. 1. $-\rho v^{2}$ as a function of $\theta$, angle between the $c$-axis and the direction of propagation of the phonon. Crosses : Brillouin scattering measurements. Points : Ultrasonic measurements. The continuous line is calculated from equation (2) using the best value of $C_{13}$.

where $\|$ and $\perp$ indicate directions parallel and perpendicular to the $c$-axis respectively. The volume compressibility is thus :

$$
\chi=\chi_{\|}+2 \chi_{\perp}
$$

Using our values for the elastic constants we obtain the values given in table II which agree well with measured compressibilities [1].

Considering the success in describing the static compressibilities of $\mathrm{GaS}$ in term of the elastic constants we proceeded to do the same for the sister compounds GaSe and InSe. These materials have isostructural layers and differ only in the stacking of the layers. Hence they are expected to behave in a very similar fashion. Using the $C_{i j}$ of GaSe [6] and InSe [7] we obtain the

Table II. - Compressibilities in units of $10^{-3} \mathrm{GPa}^{-1}$ for the three similar layer compounds, obtained from the elastic constants.

\begin{tabular}{lccc} 
& $\chi_{\|}$ & \multicolumn{1}{c}{$\chi_{\perp}$} & $\frac{\chi}{-}$ \\
$\mathrm{GaS}$ & 23.6 & 4.7 & 33 \\
$\mathrm{GaSe}$ & 24.9 & 5 & 34.9 \\
InSe & 23.7 & $1.4\left({ }^{*}\right)$ & 26.5
\end{tabular}

(*) Doubtful, see text. 
compressibilities given in table II. As can be seen $\chi_{\|}$is very similar for the three crystals. $\chi_{\perp}$ however is somewhat smaller in InSe than in the other two. This may be due to the large value of $C_{13}$ which is claimed [7] to be $32 \mathrm{GPa}$, in contrast to $12.6 \mathrm{GPa}$ for $\mathrm{GaSe}$ and $12 \mathrm{GPa}$ in $\mathrm{GaS}$.

In the $\mathrm{GaS}, \mathrm{GaSe}$, InSe series, the frequencies of corresponding zone centre modes $[8,9,10]$ are, within a few percent, in the ratio of the relevant reduced masses. Therefore, restoring forces should be quite similar and thus analogous bond strengths and compressibilities are expected among them. For this reason, this value of $\chi_{\perp}$ for InSe appear to be anomalous and more ultrasonic measurements, or direct evaluation of $\chi_{\perp}$ are probably worthwhile.

With reference to the values of the compressibilities for GaSe given in table II we should point out that they have also been determined experimentally by an undescribed method [11] which yielded $\chi_{\|}=7, \chi_{\perp}=3$ and $\chi=13$ in units of $10^{-3} \mathrm{GPa}$. These values are so far from the other values in table II that we feel they are not reliable.

4. Conclusion. - Values of the elastic constants of GaS have been obtained that do not show complete agreement with previous determinations. Because we have confirmed our results by neutron scattering, ultrasonics, Brillouin scattering and direct high pressure measurements, we feel that they are unequivocal. The linear compressibilities calculated from the values of the elastic moduli agree well with experimental data.

Contrary to what is found if the experimental values for GaSe are used, a comparison of the compressibilities calculated from the elastic constants of the sister compounds $\mathrm{GaS}$, GaSe and InSe show a remarkable but expected similarity.

\section{References}

[1] Polian, A., Besson, J. M., Grimsditch, M. and Vogt, H., Phys. Rev. B 25 (1982) 15.

[2] Powell, B. M., Jandl, S., Brebner, J. L. and Levy, F., J. Phys. C 10 (1977) 3039.

[3] Hamaguchi, C., Wasa, K. and Yamawaki, M., Proc. 3rd Inter. Conf. on Phonon Scattering in Condensed Matter, Providence (1979) Ed. by H. J. Maris (Plenum Press) 1980, p. 441.

[4] Polian, A., Kunc, K. and Rossat-Mignod, J., Proc. Inter. Conf. on Phonon Scattering, Bloomington (1981), Ed. by W. E. Bron, J. Physique Colloq. 42 (1981) C6-295.

[5] It should be noted that, in the value of $\beta$ given in reference [2] a factor $10^{12}$ has been omitted.

[6] Chiang, T. C., Dumas, J. and Shen, Y. R., Solid State Commun. 28 (1978) 173.

[7] Iskender-Zade, Z. A., Faradzev, V. D. and Agaev, A. I., Fiz. Tverd. Tela 19 (1977) 851 (Sov. Phys. Solid State 19 (1977) 492).

[8] Wieting, T. J. and Verble, J. L., Phys. Rev. B 5 (1972) 1473.

[9] Hayek, M., Brafman, O. and Lieth, R. M. A., Phys. Rev. B 8 (1973) 2772.

[10] Jandl, S. and Carlone, C., Solid State Commun. 25 (1978) 5.

[11] Aliev, N. G., Kerimov, I. G., Kurbanov, M. M. and Mamedov, T. A., Fiz. Tverd. Tela 14 (1972) 1522 (Sov. Phys. Solid State 14 (1972) 1304).

[12] BaumanN, T., Private communication. 